\title{
Irreversible Deformation of Micron-Thick Single-Crystal Silicon in the Temperature Range of $350-500{ }^{\circ} \mathrm{C}$
}

\author{
Kazuo Sato ${ }^{* \dagger}{ }^{* \dagger}$ Akira Sugimoto, and Tomo-omi Nishimura \\ Department of Micro-Nano Systems Engineering, Nagoya University, \\ Furo-cho, Chikusa-ku, Nagoya 464-8603, Japan
}

(Received July 21, 2015; accepted August 31, 2015)

Keywords: single-crystal silicon, plastic deformation, brittle-to-ductile transition, size effect, MEMS, temperature

A 2- $\mu \mathrm{m}$-thick single-crystal silicon (SCS) beam showed plastic deformation in the temperature range of $350-500{ }^{\circ} \mathrm{C}$. SCS beams made from a top layer of a silicon-on-insulator (SOI) wafer were first elastically buckled by a compressive force applied from the free end in the longitudinal direction. While maintaining the buckled state, the beams were heated to an elevated temperature for $1 \mathrm{~h}$ in air. After cooling and releasing the applied force, the residual bent profile was measured using a laser profiler. A plastic hinge was observed to appear for the beams bent in the temperature range of $350-500{ }^{\circ} \mathrm{C}$ but not at $300{ }^{\circ} \mathrm{C}$. Plasticity appeared under a large elastic strain in the range of $2-5 \%$ at the elevated environmental temperatures. Slip lines of the crystal were clearly observed at the plastically bent beam surfaces. We further demonstrated that a permanent out-of-plane deformation was obtained by applying hot air flow to a SCS film structure.

\section{Introduction}

It was conventionally believed that single-crystal silicon (SCS) is brittle below $600{ }^{\circ} \mathrm{C}$. Creep deformation was investigated above this brittle-to-ductile transition (BDT) temperature using millimeter-sized specimens. ${ }^{(1,2)}$ On the other hand, silicon nanowire was reported to show ductile fracture even at room temperature under tensile stress.(3) A 100-nm-thick silicon beam showed a trace of crystal lattice slippage on the surface, when it was bent at temperatures in the range of 100-300 ${ }^{\circ} \mathrm{C} .{ }^{(4)}$ However, in the micron range, where most of the MEMS structures are, little was reported on the $\mathrm{BDT}$ as a function of size and temperature.

In our previous reports, ${ }^{(5-7)}$ we made a series of tensile tests using SCS films in the temperature range of -50 to $500{ }^{\circ} \mathrm{C}$ and in the thickness range of submicrons to $4 \mu \mathrm{m}$. Those results are summarized in Fig. 1 in comparison with previous results. Difference in ductility is plotted by the difference in color: ductile in white, brittle in black, and less brittle in gray. Nakao et al.$^{(5)}$ reported the ductile fracture of a 4- $\mu \mathrm{m}$-thick film at $500{ }^{\circ} \mathrm{C}$. Pure brittle fracture occurred below $60{ }^{\circ} \mathrm{C}$ with a film $4 \mu \mathrm{m}$ thick, ${ }^{(6)}$ and below $-50{ }^{\circ} \mathrm{C}$ with a film $1 \mu \mathrm{m}$ thick. ${ }^{(7)}$ However, for data points colored in gray, it was unclear if irreversible plastic deformation occurs in a MEMS microstructure. We newly added experimental results with a $2-\mu \mathrm{m}$-thick SCS film in the temperature range of $350-500{ }^{\circ} \mathrm{C}$ in the figure.

${ }^{*}$ Corresponding author: e-mail: sato@aitech.ac.jp

Current address: Department of Mechanical Engineering, Aichi Institute of Technology, 1247 Yachigusa, Yakusa-cho, Toyota 470-0392, Japan 


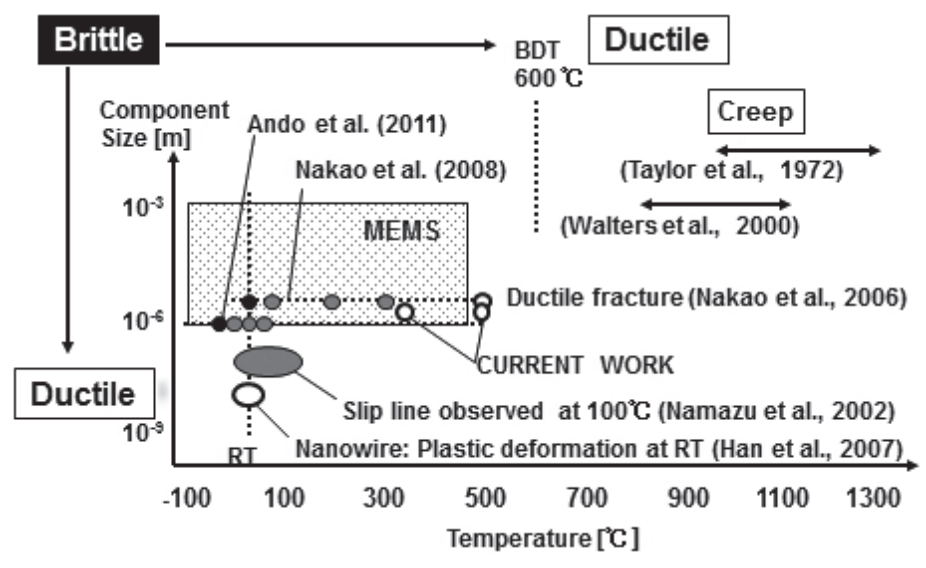

Fig. 1. BDT in relation to temperature and component size. On the basis of previous publications, dots are plotted showing ductile regions differentiated by colors: black dots for brittle, blank for ductile, and gray for intermediate. Our current work has added two ductile dots in the graph.

In this work, we switched from tensile test to bending test, aiming at applying higher stresses to a very narrow area of the silicon beam than those in the tensile test. In the case of the tensile test, specimens having a long uniform stress region tend to fracture at lower stress levels than in the bending tests. This is due to the fact that fracture probability increases according to the increase in specimen surface area and volume loaded at maximum stress during the tensile test. On the other hand, in the case of the bending test, the area of maximum stress is quite limited over the specimen. We applied large elastic strains of $2-5 \%$ to the bending specimen surface and maintained them at different temperatures. After releasing external force, we measured the beam profile and examined if irreversible deformation is observed.

\section{Experiments}

\subsection{Specimens}

SCS cantilever specimens are designed to be fabricated in an arrayed manner as shown in Fig. 2. The cantilever dimensions are 1.8-2.2 $\mu \mathrm{m}$ thick, $20 \mu \mathrm{m}$ wide, and $100 \mu \mathrm{m}$ long. They are suspended over a long cavity between the left (A) and right (B) parts of a rigid substrate. The plates $A$ and $B$ in the figure are connected to each other by flexure springs, and the spacing between the plates is closed by applying external force in the lateral direction. The beams are buckled when plate B moves toward plate A. Elastic strain in the range of 2-5\% was applied to the cantilever surface utilizing the buckling of the beam due to axial compression.

The fabrication of the specimen and a photo of the fabricated test chip are shown in Fig. 3. We used a 4-inch silicon-on-insulator (SOI) wafer as a starting material whose top silicon layer was 5 $\mu \mathrm{m}$ thick having a (100) surface orientation. It had a buffered oxide layer of $1 \mu \mathrm{m}$ thickness and a (100) substrate of $400 \mu \mathrm{m}$ thickness. The process steps are as follows.

(1) Deep reactive-ion etching (DRIE) was applied to the top silicon layer, patterning the specimen profile to its total depth of $5 \mu \mathrm{m}$. The cantilever-beam length direction was aligned to $\mathrm{Si}<110>$. 


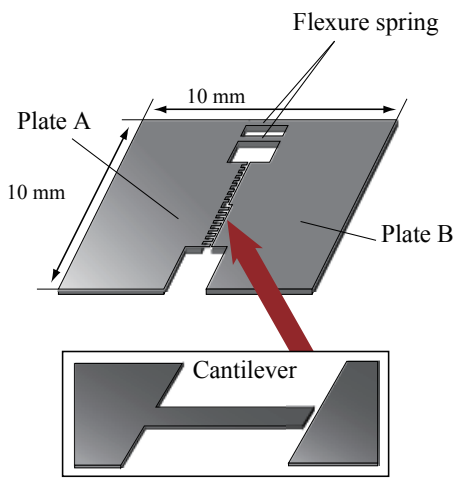

Fig. 2. (Color online) A schematic view of a test chip having an array of cantilever beams. The beams are to be buckled by a lateral force applied via plate B moving toward plate A, both connected to each other by flexure springs.

i) 4-inch SOI (100) wafer

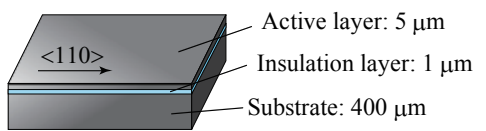

ii ) DRIE etch from top

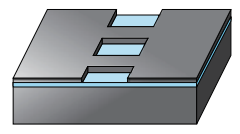

iii ) Wet etch from rear side

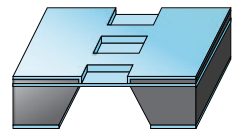

iv ) Beam thinning and splitting one end

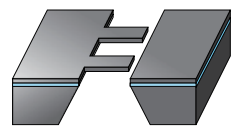

(a)

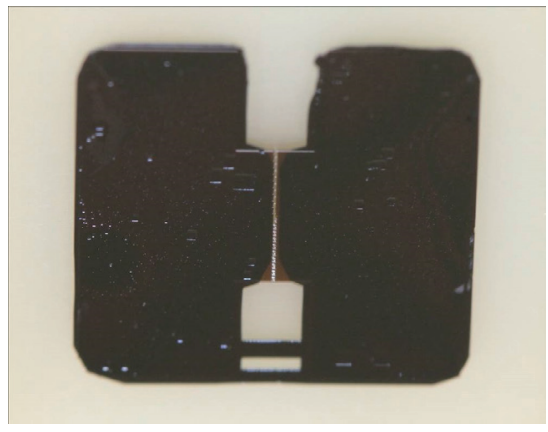

(b)

Fig. 3. (Color online) Fabrication of test specimen on an SOI wafer (a), and a photo of a fabricated test chip (b). Specimen beams are extending over a spacing between the plates A and B in Fig. 2. The spacing is visible in the photo as a vertical white-colored line between the two plates.

(2) The wafer surface was thermally oxidized to form a protective $\mathrm{SiO}_{2}$ film against alkaline etching followed by this process.

(3) A cavity was etched from the rear side of the substrate for the total thickness of $400 \mu \mathrm{m}$ by using a $20 \%$ tetramethylammonium hydroxide (TMAH) water solution containing 1 vol.\% Triton-X 100 as a surfactant.

(4) The buffered oxide layer of the SOI wafer was removed by using a buffered HF solution. A thermally grown oxide film on the top silicon layer was also etched off by this process. 
(5) The specimen thickness was decreased to a final thickness of $2 \mu \mathrm{m}$ starting from $5 \mu \mathrm{m}$. This was carried out by immersing the chip in the TMAH solution whose components are the same as that used in step (3), without agitation of the solution. ${ }^{(8)}$ The above solution is the best to maintain the uniformity of the cross-sectional shape of the SCS beam in the length direction. The thickness can be tuned by repeated cycles of etching and thickness measurement by scanning electron microscopy (SEM).

(6) Finally, one end of the doubly clamped SCS beam was cut by a focused ion-beam machining system using Ga ion as a source. Thus, an array of cantilever specimens was made on a silicon chip.

\subsection{Loading method}

Beam specimens were elastically buckled by closing the spacing between two rigid parts of the test chip. The spacing was manually closed on a fixation jig shown in Fig. 4. One side of the chip (plate A in Fig. 2) was first fixed to the jig by a clamping plate using a screw, and the other side of the chip was mechanically positioned so that buckling occurred. The buckling of the specimens was observed under an optical microscope. When beam deflection due to buckling is confirmed, the other side of the specimen chip (plate B in Fig. 2) was fixed using another clamp plate with a screw. The fixation jig was all made of SCS plates to minimize thermal expansion mismatch between the specimen and the jig, because the temperature is increased to $500{ }^{\circ} \mathrm{C}$.

\subsection{Measurement of elastic/plastic deformation}

When the specimens were fixed on the jig, the buckling profile was measured using the optical surface profiler ZYGO NewView 6200. We calculated the curvature from the deflection profile distributed along the cantilever length. Specimens were placed in an oven at an elevated temperature in air as fixed on the jig. After maintaining the strained specimen at a constant temperature for $1 \mathrm{~h}$, the specimens were removed from the oven. By comparing the cantilever's profiles before and after loading, we calculated the remaining strain, i.e., irreversible plastic deformation.

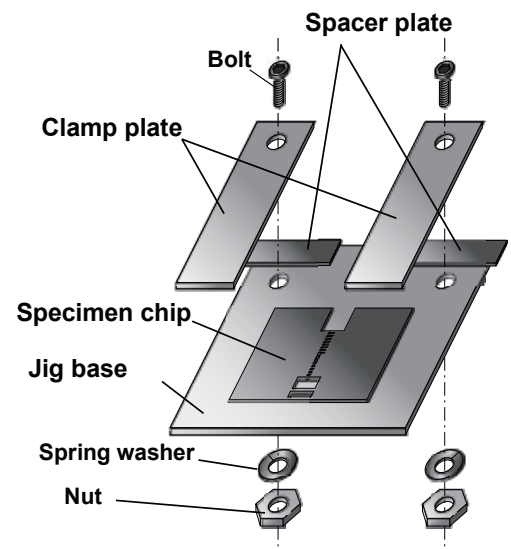

(a)

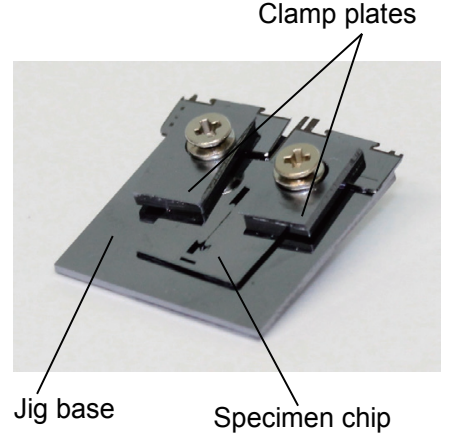

(b)

Fig. 4. (Color online) Specimen fixation jig all made of silicon plates (a) and a specimen chip fixed on the jig (b). 


\section{Results}

\subsection{Straight beam buckling test}

Figure 5 shows an array of cantilevers in an elastically buckled state. In the photo, three beams were buckled upward and another downward.

Figure 6 compares the cantilevers before (a) and after heating at $500{ }^{\circ} \mathrm{C}$ for $1 \mathrm{~h}$ and releasing the applied force (b). The cantilever after heating showed irreversible deformation at two sections on the beam, i.e., at the base in the left and in the middle of the cantilever. The irreversible deformation was relatively large and localized on the beam, as shown in Fig. 6(c), which is an

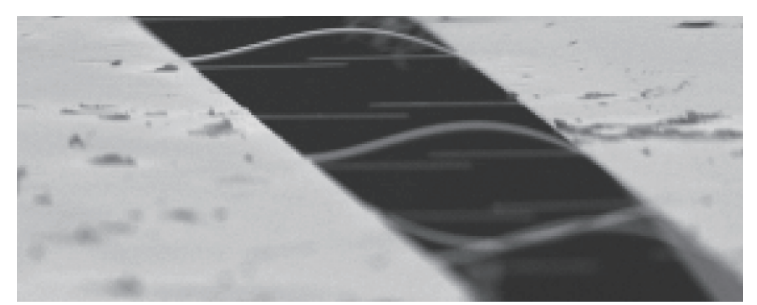

Fig. 5. Buckled cantilever beams. They have a fixed end in the left, and a free end in the right. The plate in the right is pushing the free ends of the beams simultaneously. Three beams are deflected upward, another is downward.

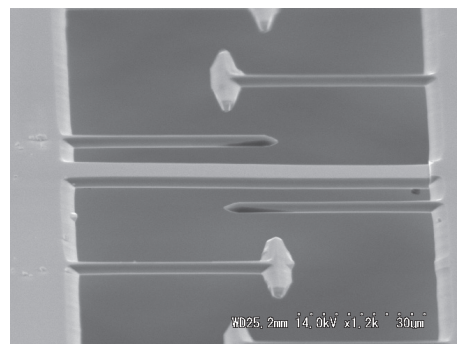

(a)

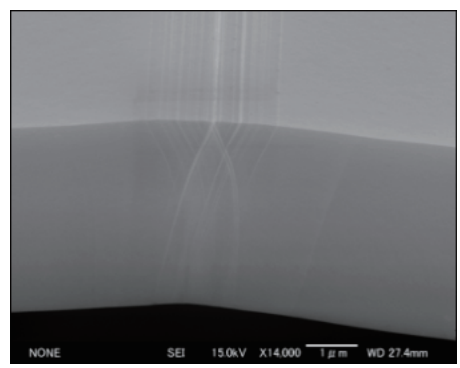

(c)

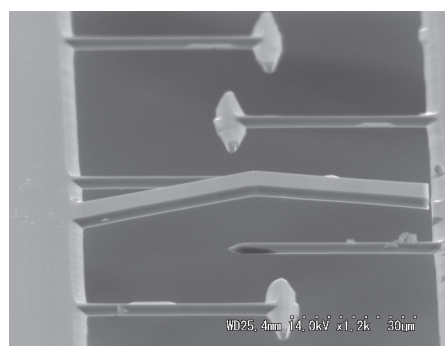

(b)

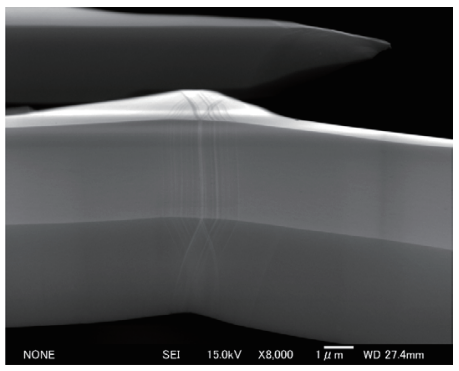

(d)

Fig. 6. SEM photos of the cantilever beam before loading (a) and after loading at $500{ }^{\circ} \mathrm{C}$ (b). The beam (b) showed plastic deformation in two narrow sections at the base and in the middle. Below are enlarged views of the deformed section in the middle of the beam, showing crystal slip lines on the top and side surfaces (c), and significant bulging at the bottom surface (d). 
enlarged view of the middle of the cantilever beam. A heavy side bulge at the bottom of the bent portion was observed, as shown in Fig. 6(d). This is due to a severe compressive plastic strain on the bottom surface of the cantilever beam.

Crystal slip lines are visible in Fig. 6(c) as white-colored stripes on the top surface, while they run crosswise on the side surface. The slip lines on the top surface were perpendicular to the cantilever length direction $<110>$ on $\mathrm{Si}(001)$. They were inclined on the side surface in Fig. 6(c). The inclined angle against the top surface was about 55 degrees, indicating that the slippage occurred on $\mathrm{Si}$ (111) near the top surface. However, when slip lines went toward the bottom of the beam, the inclined angle was difficult to identify because they became curved and faded as seen in the photo. This seems attributed partly to a nonsymmetric cross section between the top and bottom of the beam, and also to the side-bulge deformation [Fig. 6(d)] resulting in a mixed slippage system near the bottom.

A pair of short cantilever beams standing straight beside each buckled beam in Figs. 6(a) and 6(b) was for protecting the main beam during fabrication. When removing the Si substrate under the main beam by wet etching, the remaining freestanding box oxide of the SOI was easy to fracture. The fracture of the oxide film can easily propagate across the Si main beam. The shorter silicon beams were effective in protecting the main beams from the crack propagation during fabrication.

We further measured the top surface profile of the cantilever beam at room temperature in an elastically buckled state, and that after heating and releasing the load. Measurements were carried out using the optical surface profiler ZYGO NewView 6200. Figures 7(a) and 7(b) compare the

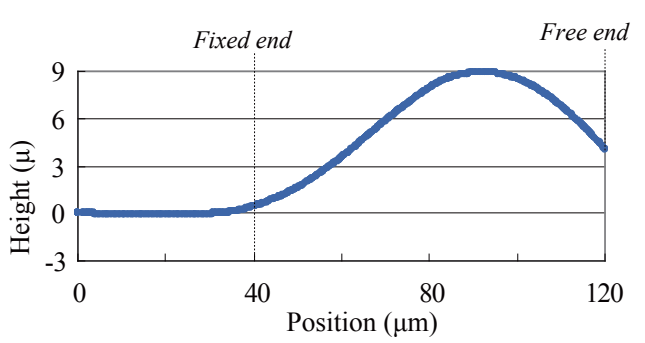

(a)

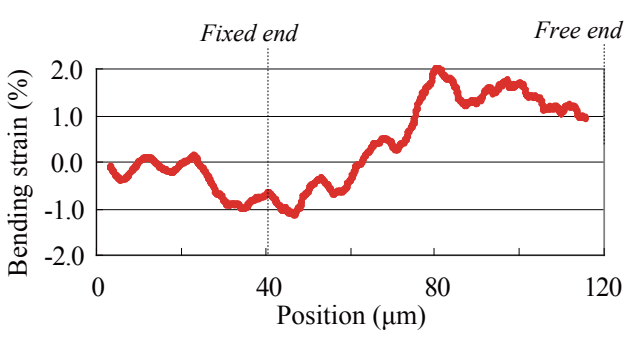

(c)

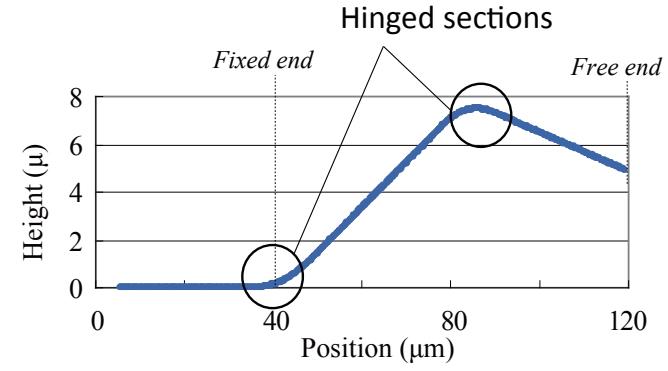

(b)

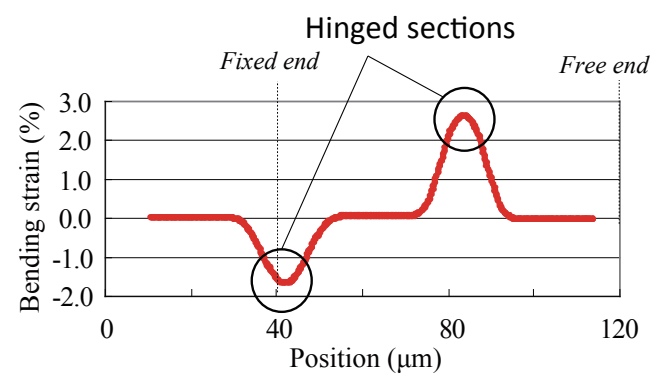

(d)

Fig. 7. (Color online) Beam profiles of elastically buckled state (a) and after heating at $500{ }^{\circ} \mathrm{C}$, cooling to RT, and unloading (b). The surface strain distributions (c) and (d) were calculated from the profiles (a) and (b), respectively. 
measured beam profiles at room temperature in the buckled state and after heat treatment at $500{ }^{\circ} \mathrm{C}$. Figures 7(c) and 7(d) show surface strain distributions for beams (a) and (b), respectively.

When the beam was loaded from the right end at room temperature, the beam buckled upward as shown in Fig. 7(a). The beam was completely in an elastic state, because, when it was unloaded at room temperature, the profile became perfectly straight, the same as in the initial state. The strain of the top surface was calculated from the beam profile. The curvature $\rho$ of the bend was calculated by differentiating the profile twice with respect to the $x$-coordinate, i.e., the length direction of the beam. Surface strain was further calculated by multiplying the curvature $\rho$ by half of the beam thickness. The surface strain distribution of the buckled beam (a) is plotted in (c) in the figure. Maximum surface strain reached $2 \%$ in tension in the middle, while surface strain was in compression $-1 \%$ near the fixed end. The surface strain was propagating to the left beyond the fixed end $(x=40 \mu \mathrm{m})$. This is due to the fact that cantilevers were fixed to a thin film of the same thickness suspended over a cavity of the substrate.

In Fig. 7(c), the bending strain distribution curve was noisy, showing a zigzag line. This was observed often for beams during loading but disappeared after unloading of the same beam. The strain profiles were calculated from the measured bent profile using a data set of nearest three points. The observed fluctuation did not decrease even we changed the distances of the three points. We think that the fluctuation is attributed to the beam stiffness fluctuation in the length direction. The beam thickness can fluctuate during beam thinning and etching from 5 to $2 \mu \mathrm{m}$ in the final process of the beam fabrication shown in Fig. 3. When the beam thickness varies by $100 \mathrm{~nm}$ with a nominally $2-\mu \mathrm{m}$-thick beam for example, the stiffness change is estimated to be $15 \%$, which is sufficient to show the zigzag line.

When the beam was heated at $500{ }^{\circ} \mathrm{C}$, cooled to room temperature (RT), and unloaded, we observed an irreversible profile change, as shown in Fig. 7(b). This is the so-called "plastic hinge" in structure mechanics, where plastic deformation is localized in a limited section of the beam. The surface strain distribution calculated from profile (b) is plotted in (d). A section between two plastic hinges showed no plastic deformation.

The same type of experiments were performed at much lower temperatures $\left(350\right.$ and $\left.300{ }^{\circ} \mathrm{C}\right)$, while the heating duration was $1 \mathrm{~h}$, the same as in the above experiment. A similar but a minimal plastic hinge was observed for a cantilever bent at $350^{\circ} \mathrm{C}$, as shown in Fig. 8(b). Figure 8(a) shows

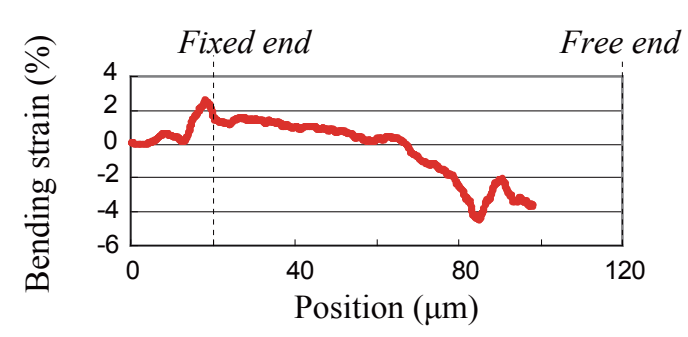

(a)

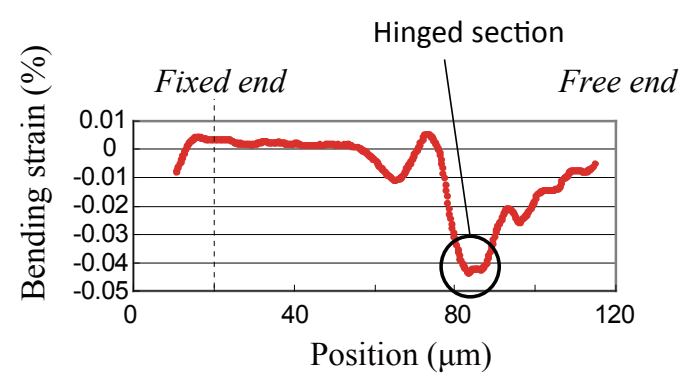

(b)

Fig. 8. (Color online) Surface strain distribution of the cantilever at elastically buckled state (a) and after thermal process at $350^{\circ} \mathrm{C}$, cooling, and unloading (b). 
the surface strain distribution along the cantilever buckled at room temperature. In this case, the beam was bent downward. Thus, the maximum surface strain was compressive $-4 \%$ in the middle of the beam, while tensile strain was $+2 \%$ near the fixed end of the beam. After heat treatment, the cantilever showed a very small residual strain of $-0.04 \%$, as shown in Fig. 6(b). This also is a local hingelike deformation due to the plasticity.

On the other hand, there was no plastic deformation observed for the cantilever beams bent at $300{ }^{\circ} \mathrm{C}$. There were no hinges left on the cantilever. Although a very small but detectable deflection was observed uniformly along the cantilever profile after the heat treatment at $300{ }^{\circ} \mathrm{C}$ and unloading, the beam profile became straight again after exposing the surface to a buffered $\mathrm{HF}$ solution and rinsing.

\subsection{Out-of-plane deformation of a SCS microstructure}

We made a spiral-shaped SCS microstructure freestanding on a SOI wafer. The 2D pattern of the spiral is shown in Fig. 9. The fabrication process steps are the same as in the straight cantilever beam experiments reported in the previous section, except the thickness of the spiral structure. Spiral film thickness was $5 \mu \mathrm{m}$ without thinning the top layer of the SOI wafer.

To demonstrate the plasticity of such a thin-film microstructure, we used hot air for blowing the spiral structure from the rear side of the SOI wafer. We used a hot air gun, HAKKO 882, whose air temperature is up to $500{ }^{\circ} \mathrm{C}$. The hot air came out from a nozzle whose inner diameter is $10 \mathrm{~mm}$. The air-flow velocity was $6 \mathrm{~m} / \mathrm{s}$. The specimen chip was fixed at a distance of $5 \mathrm{~mm}$ apart from the outlet of the blower nozzle. The spiral structure was elastically lifted upward by the air flow resulting in a large out-of-plane displacement from the chip surface. The spiral spring was exposed to the air flow of $500{ }^{\circ} \mathrm{C}$ for $30 \mathrm{~min}$. The air temperature was calibrated by using a thermocouple at the same distance from the nozzle outlet.

Figure 10 shows an out-of-plane structure of SCS. The spiral spring was permanently deformed by the air flow of $500{ }^{\circ} \mathrm{C}$ for $30 \mathrm{~min}$. Plastic deformation was observed at square corners of the spiral, and the straight arm portions remained straight.

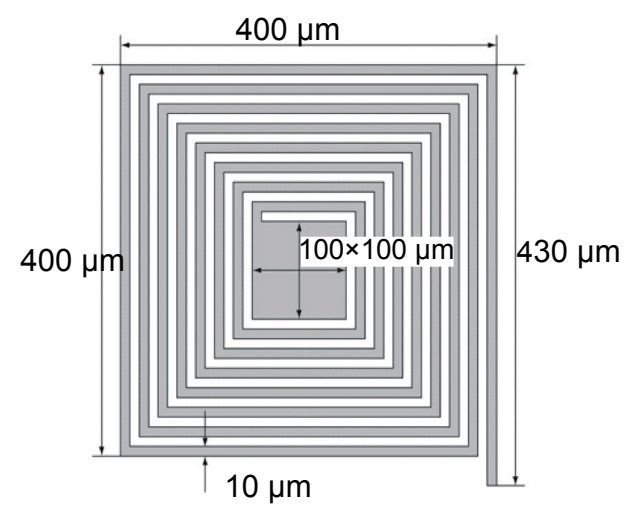

Fig. 9. Dimensions of the spiral-shaped SCS film structure. 


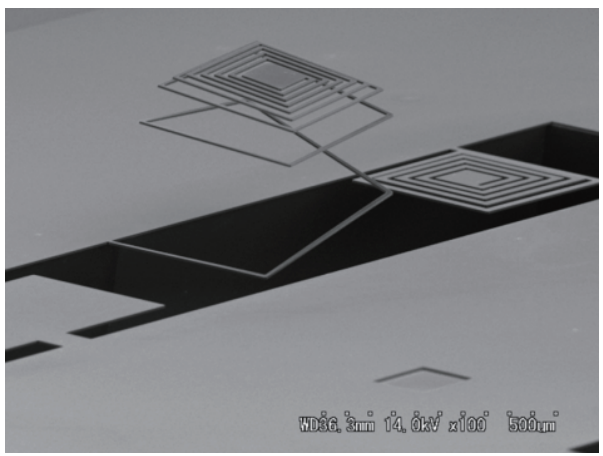

(a)

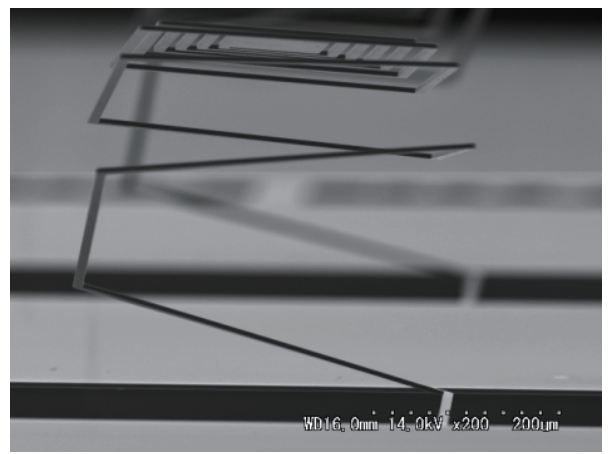

(b)

Fig. 10. An out-of-plane spiral structure of SCS. The spiral spring was permanently deformed by the air flow of $500{ }^{\circ} \mathrm{C}$ blown from the rear side of the wafer for $30 \mathrm{~min}$. Both photos [(a) and (b)] are taken for the same spiral from different angles.

\section{Discussion}

Results of our bending-type experiments for SCS cantilevers allowed us to add new plots in Fig. 1 , by making a new zone of the ductile region in relation to the component size and environmental temperature. For the specimen thickness of $2 \pm 0.2 \mu \mathrm{m}$, ductility was observed at 350 and $500{ }^{\circ} \mathrm{C}$.

BDT used to be considered as existing at around $600{ }^{\circ} \mathrm{C}$ for bulk silicon. In the case of $\mathrm{Si}$ nanowire, plastic deformation exists at room temperature. This is explained by a decrease in Peierls force due to the reduction in mechanical constraint by a reduced number of surrounding atoms around a dislocation. When a dislocation is located near the surface typically on the order of $10 \mathrm{~nm}$ apart from the surface, the above explanation seems reasonable. However, for the 2- $\mu \mathrm{m}$-thick Si film, it is difficult to accept such an explanation.

We speculate that an extremely large strain achieved in our bending test is the reason for the decrease in BDT temperature. It is known that the fracture stress of silicon is strongly dependent on the size of the structure, i.e., specimen size. A smaller specimen shows a higher fracture stress/strain. . $^{(9,10)}$ This is due to the decrease in the probability of a fracture source existing in a highly stressed region on a specimen. This is why fracture stress measured by a tensile test is usually lower than that measured by a bending test. In our bending test, the maximum elastic strain typically reached 2-5\%. Under such a highly strained condition, the Peierls potential should become shallower, i.e., dislocations can move with less thermal energy in the micron-thick film. This could be the reason for the plastic deformation that occurred at a temperature lower than that in bulk Si. We further think that the lattice slippage system itself could be affected under severe elastic strain. Molecular dynamics simulation becomes capable of analyzing the activation energies of dislocation nucleation under different conditions. ${ }^{(11)}$ We expect that molecular dynamics could explain the decrease in BDT temperature under a severely strained Si crystal of micron thickness in the near future. 


\section{Conclusion}

We investigated the ductility of thin-film SCS microstructures $2-5 \mu \mathrm{m}$ thick in the temperature range of $300-500{ }^{\circ} \mathrm{C}$. We found irreversible plastic deformation on a cantilever beam, which was first elastically buckled and exposed in the temperature range of $350-500{ }^{\circ} \mathrm{C}$. On the other hand, no irreversible deformation was observed at $300{ }^{\circ} \mathrm{C}$. We concluded that ductility appears from 350 ${ }^{\circ} \mathrm{C}$ and above for films of this size.

Our results show that SCS plastically deforms even at a postprocessing temperature of MEMS wafers, such as that of anodic bonding process. We further demonstrated the SCS ductility can be utilized for making an out-of-plane structure on a silicon wafer.

\section{References}

1 T. A. Taylor and C. R. Barrett: Mater. Sci. Eng. 10 (1972) 93.

2 D. S. Walters and S. M. Spearing: Scripta Mater. 42 (2000) 769.

3 X. Han, K. Zheng, Y. F. Zhang, X. Zhang, Z. Zhang, and Z. L. Wang: Adv. Mater. 19 (2007) 2112.

4 T. Namazu, Y. Isono, and T. Tanaka: J. Microelectromech. Syst. 11 (2002) 125.

5 S. Nakao, T. Ando, M. Shikida, and K. Sato: J. Micromech. Microeng. 16 (2006) 715.

6 S. Nakao, T. Ando, M. Shikida, and K. Sato: J. Micromech. Microeng. 18 (2008) 015026.

7 T. Ando, T. Takumi, S. Nozue, and K. Sato: Proc. IEEE MEMS (Cancun, Mexico, Jan. 23-27, 2011) p. 436.

8 P. Pal, M. A. Gosalvez, and K. Sato: J. Micromech. Microeng. 22 (2012) 065013.

9 T. Namazu, Y. Isono, and T. Tanaka: J. Microelectromech. Syst. 9 (2000) 450.

10 T. Tsuchiya, O. Tabata, J. Sakata, and Y. Taga: J. Microelectromech. Syst. 7 (1998) 106.

11 K. Shima, S. Izumi, and S. Sakai: J. Appl. Phys. 108 (2010) 063504. 